\title{
IMPACT OF DIFFERENT SELECTION STRATEGIES ON PERFORMANCE OF GA BASED INFORMATION RETRIEVAL
}

\author{
Anubha Jain ${ }^{1}$ and Swati V. Chande ${ }^{2}$ \\ ${ }^{1}$ Department of Computer Science \& IT, The IIS University, Jaipur, India and \\ ${ }^{2}$ Department of Computer Science, International School of Informatics \& Management, \\ Jaipur, India
}

\begin{abstract}
As the information proliferates, searching for relevant information has become a primary task. Searching or Information retrieval (IR) aims to help the users in organising as well as retrieving those documents from the documentary collection which are most likely to satisfy information needs of the user. An optimal Information Retrieval System (IRS) is one which retrieves only those documents from the document database which are pertinent to user's information needs, while excluding documents that are not relevant. Genetic Algorithm is described by higher likelihood of finding good solutions to large and complex problems of IR optimisation. The performance of Genetic Algorithm depends upon the decision of underlying operators used namely selection, crossover and mutation. A GA-based algorithm IRIGA (Information Retrieval Improvement using Genetic Algorithm) is developed to improve the performance of Information Retrieval System. This paper presents a comparison of performance of IRIGA when different selection methods are used. The results are analysed by conducting experiments keeping the rest of the GA parameters as constant and varying only the selection strategy.
\end{abstract}

\section{KEYWORDS}

Information Retrieval, Genetic Algorithm, Roulette Wheel Selection, Binary Tournament Selection.

\section{INTRODUCTION}

Information has always been a principal resource for an organisation, but the ways of storing and accessing data has noticeably transformed with innovations in technology and tools. More and more information services like archives, news services, libraries, electronic mail and management information systems have become online in order to provide prompt and easy access to users. Information-oriented activities to gather and process information effectively are increasingly being developed by the organisations. Information search is a focused activity leading to collecting data and processing it to become information. The demand for more advanced search tools that are capable to respond to the informational needs of a user within an organisation, is ongoing [37]. The focus of the researchers is on sorting out what is useful or relevant to the user from vast quantities of irrelevant material, rather than gathering information. Information retrieval (IR) resolves the problems of finding relevant documents from the document collections. The principal objective in the IR systems research is to increase the subset of retrieved relevant documents, while minimizing the collection of non-relevant documents being retrieved [1]. Different techniques have been proposed by researchers to enhance the performance of IR systems. Genetic Algorithm is one of the most prevalently used and powerful optimization technique in Information Retrieval domain [1][2][3] [4] [10][12][15]. It is well suited to problems which cannot be solved by mathematical evaluation and for which exhaustive search of the 
solution space is computationally infeasible. It utilises a population of individuals that undergo selection along with the genetic operators like mutation and recombination. The selection operator is responsible for selection of healthier or fitter chromosomes from the current generation to undergo recombination to generate next generation. Selection operator contributes significantly in improving the average quality of the population. Several selection methods have been developed, each having its advantages and limitations.

A Genetic Algorithm-based solution (IRIGA) is developed to improve IR performance. This paper discusses two popular selection methods namely roulette wheel selection and binary tournament selection employed with the GA-based solution IRIGA. The experimental results prove that the GA-based solution using tournament selection generates more qualitative results than roulette wheel selection.

The rest of the paper is structured as follows. In Section 2 and 3, a brief description of Information Retrieval and Genetic Algorithm are presented. Section 4 describes IRIGA used to improve the IR process. The results of the experiments and corresponding analysis are discussed in Section 5. Finally, conclusions are drawn in Section 6.

\section{INFORMATION RETRIEVAL}

Information retrieval is a field concerned with the structure, analysis, organization, storage, searching, and retrieval of information [5]. An Information Retrieval System (IRS) is software used for storage, document representation and search. The data collections managed by IRS contain large number of documents. The main concern of IRS is to provide easy, efficient and accurate information search with respect to user need [6]. Documents in the collection may take many forms, such as text or multimedia. There are three main components of an IRS [5][7]: documentary database, query subsystem \& the matching mechanism. The documents and the representations of their information contents is stored in the documentary database. Its indexer module automatically generates a representation for each document by extracting the document contents. The query subsystem permits the users to formulate their information needs. It includes a query language that assembles the rules to generate valid queries and procedures to select the relevant documents. The matching mechanism is responsible for retrieving relevant documents by comparing the extent to which the document representations meet with the requirements expressed in the query. The methods used for document indexing, internal document representation, query language and document-query matching depend upon theoretical Information Retrieval model underlying the system. These models find similarity of a document to the user query, in order to retrieve and rank the documents that reflect the query. The retrieved documents are ranked or ordered based upon a ranking strategy.

\section{Genetic AlgorithM}

Genetic Algorithms (GAs) are adaptive and best fitted heuristic optimization techniques based on the principles of the evolution via natural selection, employing a population of individuals that undergo selection in the presence of variation-inducing operators, such as mutation and recombination. This procedure is repeated several times, and typically leads to improved individuals [36]. It is characterized by the intrinsic parallel search mechanism and powerful global exploration capability in a high-dimensional space [8][9]. Due to its robustness and efficient search capabilities, application of GAs to intelligent IR has been a topic of research for the researchers, academicians and practitioners. 
The main phases while designing a GA are [10]:

- Coding the problem solutions

- Selecting a fitness function that can optimize the performance

- Choosing the set of parameters of selection, crossover and mutation

- Choosing initialization method and stopping criteria

Genetic Algorithm (GA) has been widely used in the Information Retrieval domain and encompasses a variety of areas like document and query representation, query reformulation and optimization, document indexing, document matching and ranking, automatic document clustering, relevance optimization, user profiles modeling for web search, web page classification, design of agents for Internet searching [11][12][13][14][15][16].

In Figure 1, a flowchart of Genetic Algorithm has been depicted. The initial population is defined as the collection of individuals that undergo changes to produce new generation. The members of each generation in the Genetic Algorithm process undergo the process of selection, recombination and mutation to form a new population, depending upon their fitness values. The dimension of the search space or the dimension of the GA is equivalent to the number of genes in each chromosome. In genetic model for IRIGA, the initial population consists of the set of documents encoded as genes. The encoding involves representing a document reference number. A fitness value attributed to each chromosome from the current population is evaluated at each generation. The query is sent to the information retrieval system and the query is matched against the chromosomes containing documents as genes for finding the set of more relevant documents. The process of crossover and mutation goes on until a better combination of relevant documents is achieved. The evolutionary process goes on up to a fixed number of generations or the best individual do not change for a number of generations.

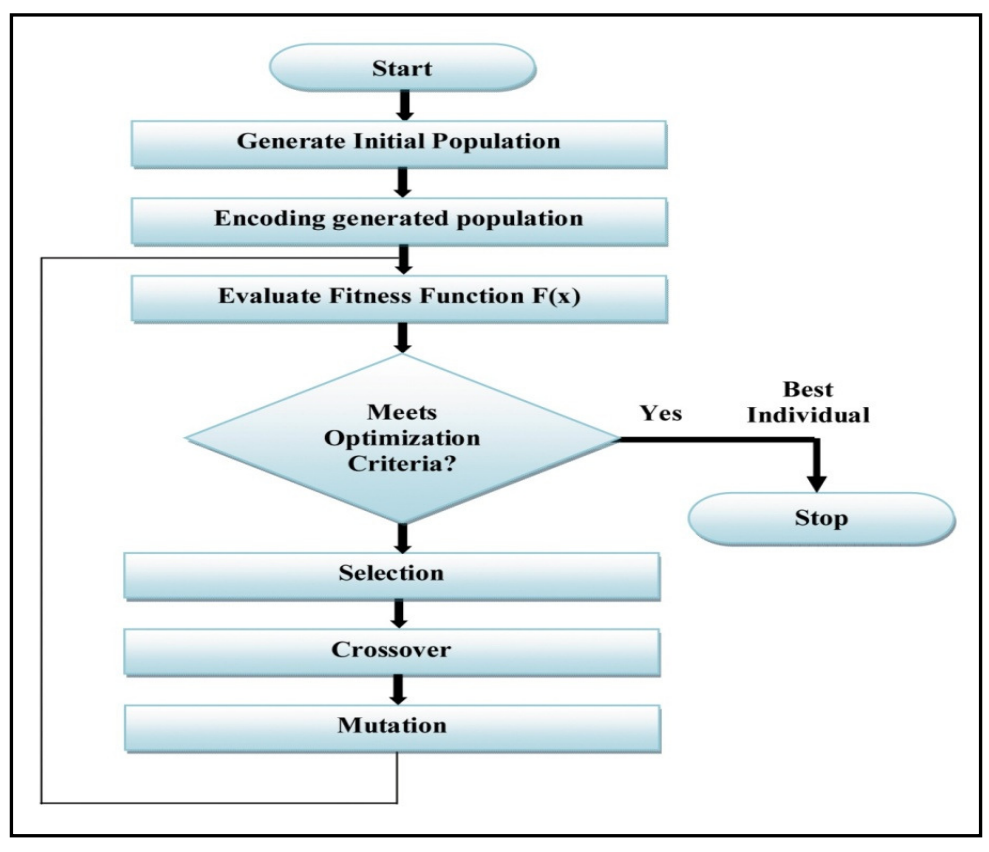

Figure1. Genetic Algorithm Flowchart 


\section{THE IRIGA}

The IRIGA (Information Retrieval Improvement using Genetic Algorithm) comprises of three modules: preprocessing, genetic algorithm and ranking. The preprocessing module performs a sequence of operations to convert the documents into a meaningful set of keywords and represent them in a manner that aids the process of finding relevant documents. The GA module helps in finding good answers to the query expressed by the user, by employing improved GA and to retrieve more of the relevant set of documents. The Genetic Algorithm module yields an optimal chromosome which represents the set of most relevant documents for that query. The ranking module arranges the retrieved documents in the order of their ranks. In this paper, the focus will be on the GA module of IRIGA, and the algorithm name will depict the GA unit. The GA module performs three genetic operations: selection, crossover and mutation. The algorithm is as follows:

Step1. Encode the given problem using integer encoding.

Step2. Create an initial population, which comprises of a specified number of chromosomes or individuals. Each individual is made up of fixed number of genes. Here, each gene represents a document from the collection.

Step3. Perform the following sub-steps until the termination criteria are satisfied:

(a)Evaluate the fitness of each chromosome or individual in the population using the fitness function.

(b)Create new population by performing following genetic operations:

(i) Selection: Select chromosomes from the current population according to their fitness value; with a given probability.

(ii) Crossover: Create two new individuals by two parents selected probabilistically from the population by recombining them at the two crossover points.

(iii) Mutation: Create a new individual by mutating an existing chromosome.

Step4. Check whether the termination condition is satisfied. If yes, the chromosome with the highest fitness value forms the result of the genetic algorithm. If not, repeat Step 3.

\subsection{Crossover}

Crossover is a genetic operator responsible for generating the offsprings from the existing population. The crossover or recombination operator combines two selected chromosomes (parents) to produce a new chromosome(s) (offspring) of the new generation. The idea behind crossover is that the new chromosome may be better than both of the parents if it takes the best characteristics from each of the parents. A chromosome with higher fitness has a better chance to be selected than the ones with lower fitness, so that good solution always continues onto the next generation [17][18].

IRIGA employs two-point crossover with the condition that each gene of a chromosome should be unique. In this crossover technique, two crossover points are chosen randomly from the parent chromosomes. The values between the chosen points are exchanged, and then a careful procedure is followed to eliminate any repeated document-ids from the solution, to produce two new 
offsprings. It is ensured that all genes are found exactly once in each chromosome and no gene is duplicated. The crossover probability for this study is taken as 0.8 .

\subsection{Mutation}

Mutation operator introduces a little amount of change in the chromosome structure at random. It guarantees that no point in the search space has a zero probability of being examined [19]. It prevents early convergence of the population and the genetic drift by changing the values of a small number of randomly selected alleles in the chromosome to continuously introduce variation. There are many objectives of applying mutation like restoring lost data, exploring variety of data [17], enhancing diversity of the solution [23] and reducing the possibility of converging to a local optimum, as opposed to the global optimum.

IRIGA uses one-point mutation wherein a single randomly selected gene gets replaced with a random value [1][14][15][18]. It has shown better performance as compared to other mutation types. The mutated gene may be of lower performance than the old gene. The mutation can disrupt the building blocks, in case the mutated gene is lower quality than the older gene. To resolve this problem, replacement of new gene with older gene is done subject to the condition that the fitness of replacing gene is same or better than the one getting replaced. The mutation probability for this research is taken as 0.7 .

\subsection{Fitness Function}

The generation process of GA depends upon the objective function being used. The objective or fitness function is like the back bone of every optimisation problem. The fitness function evaluates the performance of each individual chromosome to judge its contribution in GA. It evaluates the significance or relevance of the document to the user query. Thus selection of an appropriate fitness function is of high importance. The fitness function can either be standard fitness function based on similarity measures or customised fitness function.

The standard fitness functions are based on term weight within document or within collection as the central component. The term weight is a number or score assigned to a term reflecting its importance within a document. The term weights depend upon local factors as well as global factors. Local factors consider the term's importance within the document e.g. term frequency (tf), while global factors consider the term in context of entire documents collection e.g. document frequency (df). The similarity function is a measure of degree of closeness of document vector to the user query vector. The most popular similarity measure is cosine similarity measure, Dice coefficient, Jaccard coefficient and Inner Product [24]. These measures were developed specifically for systems implemented using vector space model. They are simple, straightforward and their results reflect high performance.

Many authors have developed their own custom fitness function to evaluate a chromosome [17][25][27][28]. They have proposed a new function or have combined existing similarity measures to evolve new fitness formula. In [17], a new fitness function was proposed as the distance between terms weights of a given chromosome and the query vector. It performed better than cosine similarity fitness function in terms of complexity and precision-recall. In [25], the authors used non-interpolated average precision as fitness function. In [26], the authors proposed their own fitness function involving several local and global terminals to search for the contents of web documents.

The customised fitness function developed for IRIGA calculates the fitness of each gene as product of two factors: proportion of the query terms that exist in the document and the 
summation of weight of each query term in the document. The fitness scores of each document that is a part of a chromosome is summed together to obtain the fitness value of a chromosome. The fitness of chromosome $c_{\mathrm{i}}$ is given as:

$$
F\left(c_{i}\right)=\sum_{l=1}^{L} f\left(d_{l}\right)
$$

where $L$ represents the length of chromosome $c_{j}$. The fitness of a gene $d_{1}, l=\{1,2, \ldots, n\}$ is given as:

$$
\mathrm{f}\left(\mathrm{d}_{\mathrm{l}}\right)=\frac{\sum_{i=1}^{n} \mathrm{y}_{\mathrm{i}}}{n} \times \sum_{i=1}^{n} w_{i}
$$

\subsection{Termination Criteria}

Besides genetic operators, termination criterion is a significant factor influencing the search process. The process of GA stops either when a fixed number of generations have been produced or a satisfactory fitness level has been reached for the population or the population has converged significantly. Widely used termination criteria are[1]:

- Reaching optimal solution (which is often difficult to recognise)

- Processing fixed number of generations [17][29]

- Processing specific number of generations without any improvement in population.

Generally, the iterative process of Genetic Algorithm is carried out for a fixed number of generations ranging from 50 generations to 1200 generations [1][17][18][30].

IRIGA uses two of these decisive factors as termination criteria. IRIGA stops either when 50 generations have been reached or when best fitness value does not improve for five successive generations.

\section{SELECTION STRATEGIES}

The selection mechanism has direct impact upon the exploitation factor in the "exploitation versus exploration" trade off [8]. The parent being selected by the selection process undergoes crossover and mutation to generate offsprings for the next generation. A fitter parent will produce fitter offspring. Thus, individuals with higher fitness have higher chances of being copied into next generation. Two selection strategies used in IRIGA are discussed in this paper.

\subsection{Roulette Wheel Selection}

The most common method is simple random sampling which performs roulette wheel selection, wherein probability of selection of each individual is proportional to its fitness [8][34]. This method results in best chromosomes achieve more copies as compared to worst ones, but suffers from high time complexity and selection of certain parents which may result in faster convergence. 
The roulette wheel selection is explained in the following steps [32]:

1) Add the fitness values of each individual in the population to compute total fitness.

2) Evaluate the relative fitness of every individual, by computing the proportion of each individual's fitness value to the total fitness of the entire population.

3) Partition a roulette according to the proportions computed in step 2. The area of the roulette sector is proportional to the individual's probability to be selected. Rotate the roulette wheel ' $n$ ' times, where $\mathrm{n}$ is the number of individuals in the population. The individual pointed by the pointer is selected.

\subsection{Tournament Selection}

Another selection method is tournament selection, where group of individuals are randomly chosen from the population and a tournament is held amongst them. The winner is put in the mating pool. The pool has higher average fitness than average population fitness, drives GA to improve the fitness of successive generations. This selection mechanism is efficient in terms of complexity and allows population diversity. It also allows few weak candidates to participate, which could be useful in following operations to yield good individuals in next generations.

The binary tournament selection is the one where two individuals are selected randomly from the population. The chromosome having higher fitness value between the two selected chromosomes is selected to be the parent. The objective of this approach is to cover wider range of values by selecting the fitter individual amongst two randomly chosen individuals in the population for the next generation. The tournament selection does not involve arithmetical computation based on the fitness value, it only compares the individuals by their fitness values [32]. Also, the time complexity of tournament selection i.e. $\mathrm{O}(\mathrm{n})$ is lesser than other selection techniques and therefore the fastest [34].

The following steps show selection using binary tournament selection strategy [32]:

1) Select two chromosomes $\mathrm{x}$ and $\mathrm{y}$ randomly to take part in the tournament.

2) Compare the two chromosomes; select the chromosome with higher fitness value.

3) Repeat Step (2) for $\mathrm{n}$ times, where $\mathrm{n}$ is the size of the population.

\section{EXPERIMENTS}

The experiment was conducted with an aim to analyse the behaviour of IRIGA under different selection schemes. The experiment was performed on a dataset of 874 documents prepared specifically for the study. The collection contains abstracts from the field of Computer science, Information technology and Management. The algorithm is tested upon 113 queries.

The performance of the IR is evaluated in terms of average 11-point recall-precision, recall and $\mathrm{P} @ \mathrm{~K}$ measure. Average precision-recall measures the precision at 11-points of recall from 0.0 to 1.0. This measure evaluates the system in terms of percentage of total relevant documents. Average precision is also calculated using precision values for all points of recall. $\mathrm{P} @ \mathrm{~K}$ measure or Precision at Rank $\mathrm{K}(\mathrm{K}=1,5,10,15,20)$ evaluates the performance of first few retrieved documents only. It is the relative value of relevant documents in the first $\mathrm{K}$ retrieved documents [13][22][35]. Recall is the ratio of relevant documents retrieved to the total number of relevant 
documents. This method evaluates the system upon the number of documents retrieved. Also, the system is evaluated upon number of generations and time taken by GA module to converge in addition to these measures.

The experiment compared the performance of IRIGA upon application of two popular selection techniques: Binary Tournament and Roulette wheel. For this experiment, the maximum number of generations is taken as 50. For each technique, the algorithm was executed for 7 independent runs on all the 113 queries of the document collection, and then the results were analysed. Table 1 shows a comparative analysis of IRIGA with different selection strategies in terms of various evaluative measures.

Table 1: Evaluation parameter values for different techniques

\begin{tabular}{|c|c|c|c|c|}
\hline $\begin{array}{c}\text { SELECTION } \\
\text { TECHNIQUE }\end{array}$ & $\begin{array}{c}\text { AVERAGE } \\
\text { CONVERGENCE } \\
\text { (in generations) }\end{array}$ & $\begin{array}{c}\text { AVERAGE } \\
\text { EXCUTION } \\
\text { TIME } \\
\text { (in seconds) }\end{array}$ & RECALL & $\begin{array}{c}\text { AVERAGE } \\
\text { PRECISION }\end{array}$ \\
\hline $\begin{array}{c}\text { Binary } \\
\text { Tournament }\end{array}$ & 38.5 & 0.7 & 0.93 & 0.57 \\
\hline $\begin{array}{c}\text { Roulette } \\
\text { Wheel }\end{array}$ & 41.4 & 0.6 & 0.87 & 0.53 \\
\hline
\end{tabular}

The table 1 shows that binary tournament with replacement converges faster than roulette wheel. The results of recall and average precision are much higher in case of binary tournament. The binary tournament technique converged on an average at 38.5 generations, while roulette wheel converged at 41.4 generations on average. This shows that former selection technique is quicker than the latter selection criterion by $7.6 \%$, and at the same time the average precision of binary tournament technique is higher by $6.4 \%$ than roulette wheel. Though average execution time taken by each query is lesser in case of roulette wheel, the retrieval results are better in case of binary tournament selection technique.

It is found that there is an overall improvement of $6.4 \%$ in average precision measure when using Binary Tournament selection technique over the Roulette Wheel selection scheme. This shows that the Binary Tournament selection scheme retrieves more relevant documents as compared to roulette wheel. Figure 2 displays \% improvement between two selection schemes graphically. The system retrieves higher percentage of relevant documents when using binary tournament selection strategy. 


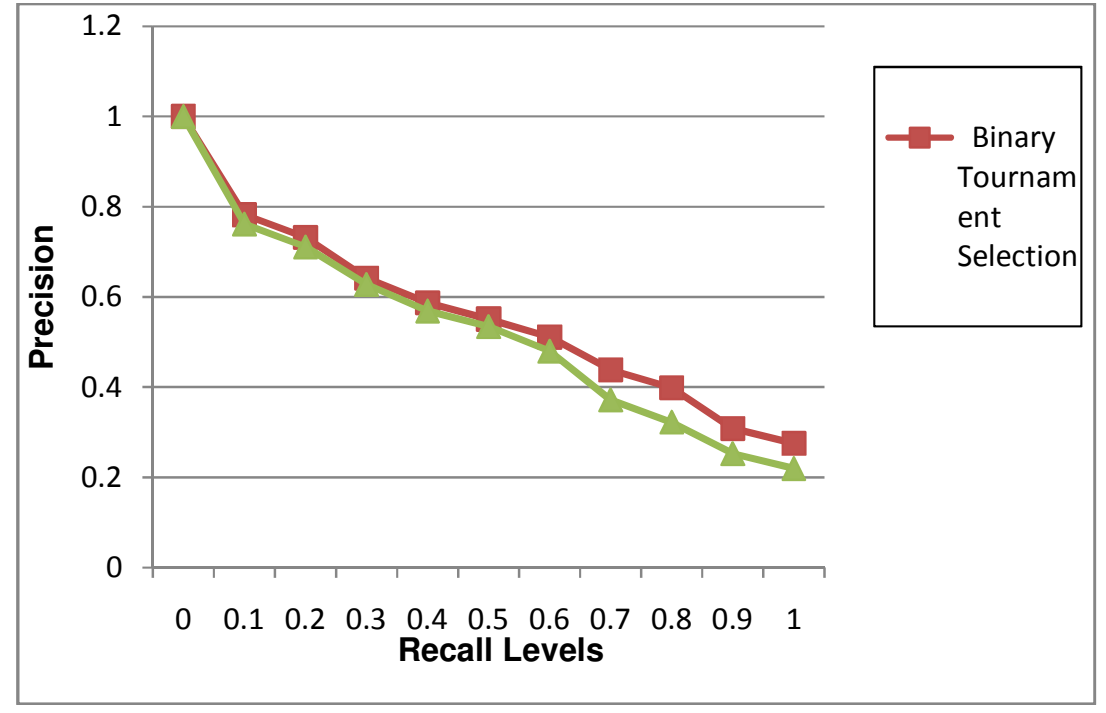

Figure 2. Comparison of Recall-Precision for different Selection Schemes

On analysing the P@K measure for the two selection schemes from Table 2, we see an improvement of $2 \%$ at first 10 documents retrieved, and an improvement of $3.1 \%$ at top-20 documents retrieved. This shows binary tournament selection retrieves more relevant documents at higher ranks. This difference is not very high, but there is an improvement of $7.1 \%$ in recall value from roulette wheel to Binary Tournament. Thus Binary Tournament yields better more relevant documents than roulette wheel.

Table 2: \% improvements in different evaluative measures for binary tournament and roulette wheel selection scheme

\begin{tabular}{|c|c|c|c|}
\hline P@K & $\begin{array}{c}\text { BINARY } \\
\text { TOURNAMENT }\end{array}$ & $\begin{array}{c}\text { ROULETTE } \\
\text { WHEEL }\end{array}$ & \% Improvement \\
\hline P@1 & 0.68 & 0.67 & $1.3 \%$ \\
\hline P@5 & 0.51 & 0.49 & $2.2 \%$ \\
\hline P@10 & 0.39 & 0.38 & $2.0 \%$ \\
\hline P@15 & 0.31 & 0.30 & $2.2 \%$ \\
\hline P@20 & 0.29 & 0.25 & $3.1 \%$ \\
\hline Recall & 0.93 & 0.87 & $7.2 \%$ \\
\hline
\end{tabular}

\section{CONCLUSION}

The GA-based algorithm IRIGA was developed to improve the retrieval performance using Genetic Algorithm. Different selection strategies are employed to study their impact on the retrieval result. The experimental results show that the performance of IRIGA based on tournament selection outperforms the one based on roulette wheel selection, both in terms of quality of results as well as the convergence speed of GA. 


\section{REFERENCES}

[1] Snásel V., A. Abraham, S.S.J. Owais, J. Platos, and P. Krömer (2009) Optimizing Information Retrieval Using Evolutionary Algorithms and Fuzzy Inference System, In Proceedings of Foundations of Computational Intelligence (4), pp.299-324, 2009.

[2] Tamine L. \& M. Boughanem (2001), Applying Heuristics to Improve a Genetic Query Optimisation Process in Information Retrieval, ECIR, Darnstad.

[3] Kim, S. and B. T. Zhang (2003), Genetic mining of HTML structures for effective web document retrieval. Applied Intelligence, 18(3), 243-256.

[4] Krömer, P., V.Snášel, J. Platoš and A. Abraham (2010), Evolutionary improvement of search queries and its parameters. In 10th IEEE International Conference on Hybrid Intelligent Systems (HIS), 147 152.

[5] Salton, G. and M. H. McGill (1983), Introduction to Modern Information Retrieval, McGraw-Hill.

[6] F. Crestani and G. Pasi (1999), Soft Information Retrieval: Applications of fuzzy set theory and neural networks. Neuro-Fuzzy Techniques for Intelligent Information Systems, pp 287-315, Springer Verlag, Heidelberg, DE.

[7] Baeza-Yates, and Ribeiro-Neto (1999), Modern Information Retrieval, Addison Wesley.

[8] Goldberg, D. E. (1989), Genetic Algorithms in Search, Optimization, and Machine Learning, Addison-Wesley, Reading, MA.

[9] Holland, J. (1992), Adaptation in Natural and Artificial Systems: An Introductory Analysis with Applications to Biology, MIT Press.

[10] Schmitt, L. M. (2001), Fundamental Study, Theory of genetic algorithms, Theoretical Computer Science, 259, 1-61.

[11] J. J Yang \& R. R Korfhage (1993), Query Optimisation in Information Retrieval Using Genetic Algorithms, ICGA.

[12] Vrajitoru, D. (1997) Genetic algorithms in information retrieval. AIDRI97: Learning; From Natural Principles to Artificial Methods.

[13] Pérez-Agüera, J. R. (2007) Using genetic algorithms for query reformulation. In BCS IRSG Symposium: Future Directions in Information Access, 100.

[14] Klabbankoh, B. and O. Pinngern (2000) Applied Genetic Algorithms In Information Retrieval. Faculty of Information Technology, King Mongkuts Institute of Techology Ladkrabang.

[15] M. O. Nassar, Feras Fares Al Mashagba, Eman Fares Al Mashagba (2013), Investigating Genetic algorithms to optimize the user query in the vector space model, Australian Journal of Basic and Applied Sciences, 7(2), pp. 47-53.

[16] Cordón, O., E. Herrera-Viedma, C. López-Pujalte, M. Luque and C. Zarco (2003), A review on the application of evolutionary computation to information retrieval. International Journal of Approximate Reasoning 34(2), 241-264.

[17] Radwan, A. A. A., B. A. A. Latef, A. M. A. Ali and O.A. Sadek (2006), Using genetic algorithm to improve information retrieval systems. World Academy of Science and Engineering Technology, 17(2), 6-13.

[18] Aly, A.A. (2007), Applying genetic algorithm in query improvement problem. International Journal Information Technologies and Knowledge 7(1), 309-316.

[19] Beasley, D., D. R. Bull and R. R. Martin (1993a), An overview of genetic algorithms: part 1. Fundamentals University Computing, 15(2), 58-69.

[20] Beasley, D., D. R. Bull and R. R. Martin (1993b), An overview of genetic algorithms: part 1. Fundamentals University Computing, 15(2), 58-69.

[21] Spears, W. M. and K.A. De Jong (1990), An Analysis of Multi-point Crossover. Naval Research Lab Washington DC.

[22] Vrajitoru, D. (1998), Crossover Improvement for the Genetic Algorithm in Information Retrieval. Information Processing and Management. 34(4), 405-415.

[23] Dianati, M., I. Song and M. Treiber (2002), An Introduction To Genetic Algorithms And Evolution Strategies, Technical report, University of Waterloo, Ontario, N2L 3G1, Canada.

[24] Safaa I. Hajeer (2012), Comparison on the Effectiveness of Different Statistical Similarity Measures, International Journal of Computer Applications (0975 - 8887), Vol 53(8), September 2012.

[25] Horng, J. T., and C.C. Yeh (2000), Applying genetic algorithms to query optimization in document retrieval. Information processing \& management. 36(5), 737-759. 
[26] Al-Dallal, A. and R. Abdulwahab (2009) Genetic Algorithm Based to Improve HTML Document Retrieval, Proceedings of Second International Conference on Developments in eSystems Engineering (DeSE'09), Abu Dhabi, UAE, 343-348.

[27] Cummins, R. and C. O'riordan (2006 a) Evolving local and global weighting schemes in information retrieval. Information Retrieval, 9(3), 311-330.

[28] Cummins, R and C. O'riordan (2006 b) A framework for the study of evolved term-weighting schemes in information retrieval. In: Stein B, Kao O (eds) TIR-06 Text based Information Retrieval, Intl. Workshop. ECAI, Riva del Garda, Italy, 6 pages.

[29] Owais, Suhail SJ, Pavel Krömer, and Vaclav Snasel (2005), "Query optimization by Genetic Algorithms." In DATESO, vol. 129, pp. 125-137.

[30] Húsek, D., V. Snásel, S.S.J. Owais and P. Krömer (2005), Using Genetic Algorithms for Boolean Queries Optimization. IMSA, 178-184.

[31] Maitah, W., M. Al-Rababaa and G. Kannan (2013), Improving the Effectiveness of Information Retrieval System Using Adaptive Genetic Algorithm. International Journal of Computer Science \& Information Technology, 5(5):91-105.

[32] Zhong, J., X. Hu, M. Gu and J. Zhang (2005) Comparison of performance between different selection strategies on simple genetic algorithms. In Computational Intelligence for Modelling, Control and Automation, 2005 and International Conference on Intelligent Agents, Web Technologies and Internet Commerce, International Conference on, 2, 1115-1121.

[33] Yang, J. J., R. Korfhage and E. M. Rasmussen (1992) Query improvement in information retrieval using genetic algorithms - A report on the experiments of the TREC project. In Proceedings of the first text retrieval conference, 31-58.

[34] Anbumani, K. and R. Nedunchezhian, (Eds.) 2010) Soft Computing Applications for Database Technologies: Techniques and Issues. IGI Global.

[35] Carlberger, J., H. Dalianis, M. Hassel and O. Knutsson (2001) Improving precision in information retrieval for Swedish using stemming. In Proceedings of NODALIDA'01 - 13th Nordic conference on computational linguistics. Uppsala, Sweden.

[36] Jain A., S. Chande and P. Tiwari (2014) Relevance of Genetic Algorithm Strategies in Query Optimization in Information Retrieval. International Journal of Computer Science and Information Technologies(IJCSIT), 5(4), 5921-5927. url:http://www.ijcsit.com/ijcsit-v5issue4.php

\section{Authors}

\section{Anubha Jain}

Anubha Jain has over seventeen years of experience in teaching, R\&D and industry. Knowing that teaching is the best form of giving knowledge back to society, she has been involved in teaching and mentoring several students in the field of Computer Science. She is currently pursuing her PhD, in the field of Information Architecture \& Information Retrieval. She is author of nine books and a number of papers at national and international level. She is associated with different academic bodies in diverse capacities including being the convener of Board of Studies, member of Academic Council, Curriculum Committees, etc.

\section{Dr. Swati V. Chande}

Prof. Swati V Chande has over 24 years of experience in teaching, industry and R\&D. She also has an extensive corporate and technical training experience. She did her M.S. in Software Systems from Birla Institute of Technology and Science, Pilani and also has a M.Sc. in Mathematics. Her research interests include Database Management, Genetic Algorithms, Computational Studies, and Software Engineering. Presently, she is supervising research work of eight doctoral scholars, and has also guided several postgraduate projects and research studies. She has authored a book and a significant number of papers and articles in national and international publications. Dr. Chande is a member of various professional bodies and is associated with different academic institutions in diverse capacities including being a member of Academic Council, Board of Studies, Departmental Research Committee, Curriculum Committees, etc. of different Universities. 